\title{
Respuesta de los autores
}

\author{
Authors' response
}

Ugalde, Antonio ${ }^{1}$; Homedes, Núria²

${ }^{1}$ Doctor en Sociología. Profesor Emeritus de Sociología, University of Texas at Austin, EE.UU. augalde@mail.utexas.edu

${ }^{2}$ Doctora en Salud Pública. Profesora, School of Public Health, University of Texas at Houston, EE.UU. nhomedes@utep.edu

Respuesta a los comentarios de: Gonorazky SE; Tajer CD; Ferrante D; Martínez RA; Gómez-Vargas M; Lorenzo C, Garrafa V; Forcades i Vila T. Salud Colectiva. 2011;7(2):149-173.

Debemos agradecer a Salud Colectiva la iniciativa de organizar este debate sobre aspectos éticos y de derechos humanos en torno a la ejecución de los ensayos clínicos en América Latina. Agradecemos igualmente la oportunidad de responder a los comentarios que han realizado ocho expertos procedentes de varios países (Argentina, Brasil, Costa Rica y España) con diferentes trayectorias profesionales (miembros de comités de ética independientes e institucionales, investigadores principales, activistas, bioeticistas, directores de agencias reguladoras, académicos, y ejecutivos de farmacéuticas trasnacionales). Esta diversidad de países y perspectivas ha añadido profundidad al debate.

Como el objetivo es buscar respuestas a los problemas con que se enfrentan los ensayos clínicos en América Latina, que por lo que se puede leer en la literatura no son muy diferentes de los que se dan en otros países de bajos y medianos ingresos, intentaremos hilvanar las ideas expuestas que consideramos más importantes y que sugieren soluciones o cuestionan las ideas que hemos ofrecido en nuestro artículo (1).

La dicotomía adicional engaño/mentira presentada por Marvin Gómez-Vargas (2) es importante porque, en definitiva, la opacidad y las barreras que el investigador externo encuentra para recabar datos, aclarar dudas y comprobar que los ensayos clínicos se están realizando de acuerdo con el protocolo y con los criterios éticos aceptados internacionalmente son tan grandes que inducen a pensar que la industria tiene algo importante que ocultar. Un primer tema a debatir es cómo se puede evitar que los gobiernos permitan que el estribillo tan frecuentemente invocado por la industria -"debemos proteger los secretos industriales"- sea una excusa para no proteger los derechos de los pacientes, tanto de los que participan en los ensayos clínicos, como de los que consumen los medicamentos. Gómez-Vargas afirma, desde su experiencia como ex ejecutivo en la industria que, con más frecuencia de la que pensamos, los ensayos clínicos tienen objetivos mercantilistas y no científicos. Teresa Forcades i Vila, al citar la investigación de Adriane Fugh-Berman sobre Wyeth (3 p.172), resalta este mismo punto que se da por supuesto en los comentarios de Ricardo Martínez (4), Claudio Lorenzo y Volnei Garrafa (5), Sergio Gonorazky (6) y Daniel Ferrante (7). Lo expuesto por estos autores y el decálogo presentado por Carlos Tajer (8), nos permiten concluir que hay consenso en la siguiente frase: si la protección de los secretos industriales implica la desprotección de los derechos humanos, es necesario buscar alternativas a la implementación de los ensayos clínicos.

El origen del problema lo han expresado con claridad y en muy pocas palabras Lorenzo y Garrafa al indicar que "...los ensayos clínicos son eminentemente una actividad industrial $y$, como todas las demás iniciativas empresariales, se 
encuentran inmersos en los juegos de poder del libre mercado" (5 p.167). Añadimos que el juego del poder es lo que lleva a engañar. Hace apenas unos días, el influyente y conservador periódico Financial Times incluía una sección sobre la corrupción de las empresas transnacionales (9). Su lectura permite afirmar que la corrupción es una práctica casi inherente a las características competitivas del capitalismo: el juego de las empresas consiste en superar a la competencia, y superar significa poder esquivar la vigilancia del Estado. Es más, según el autor, el Estado no ha demostrado un gran interés en detectar la corrupción; en el caso de EE.UU., solo recientemente, el departamento del Tesoro ha empezado a poner multas cuantiosas a las transnacionales con el fin de generar los fondos que necesita. Entre los múltiples ejemplos que cita el artículo del Financial Times no faltan los de la industria farmacéutica que, aunque produce sustancias necesarias para la salud de los humanos, se comporta igual que las industrias que producen artículos de consumo que no son esenciales para la vida. De allí la necesidad de una regulación exigente y sin compromisos por parte del Estado.

Carlos Daniel Tajer (8 p.152) afirma que: "...sería un error de iguales consecuencias negativas pensar que todo es fraude, corrupción y manipulación en la investigación clínica multicéntrica". Lamentablemente, la falta de transparencia impide verificar las dimensiones y la frecuencia del fraude. Es obvio que no se puede afirmar que todo es fraude simplemente porque la industria no permite ser estudiada, pero las respuestas de los demás comentaristas y la literatura existente, incluyendo documentos que por limitaciones de tiempo ni ellos ni nosotros hemos podido citar, permiten afirmar que no son casos excepcionales. En todos los números del Boletín Fármacos (10), hemos reproducido casos de fraude y corrupción de la industria farmacéutica, incluyendo de ensayos clínicos publicados en revistas científicas y prensa no sensacionalista.

Hay que evitar acercamientos conspiratorios, pero no se debe ocultar lo que los datos sugieren. En EE.UU. se realizan encuestas sobre la confianza de los ciudadanos en las instituciones, y los resultados no son nada favorables a la industria farmacéutica, que junto a las tabacaleras ocupan los dos lugares más bajos. Es responsabilidad de la industria desmantelar las barreras que han establecido en complicidad con las agencias reguladoras -incluyendo la Food and Drug Administration (FDA) y la Agencia Europea de Medicamentos (EMA) - en torno a cómo se realizan los ensayos clínicos. Esto facilitaría que los investigadores independientes pudieran dimensionar el fraude existente. En su contribución Gonorazky nos presenta un buen ejemplo sobre este punto cuando se refiere a los Comités de Monitoreos de Datos de Ensayos Clínicos y, si el espacio lo permitiera, se pondrían ofrecer muchos ejemplos más.

Las manipulaciones en los ensayos clínicos contribuyen a crear un alto nivel de desconfianza sobre lo que se ha llegado a Ilamar "medicina basada en la evidencia". Varios de los comentarios mencionan los fraudes en las publicaciones clínicas de mayor prestigio. ¿Es posible construir una medicina basada en la evidencia si las revistas científicas no pueden detectar los fraudes? ¿ $\mathrm{O}$ a lo mejor -como se sospecha- publican artículos que están subvencionados aunque los revisores sugieran que no se deben publicar? Tanto para los clínicos como para los ciudadanos es cada vez más difícil distinguir qué parte de la información diseminada es científica y cuál responde a intereses empresariales, políticos, ideológicos o incluso a las ambiciones personales de algunos investigadores con poca conciencia.

Como indica Tajer (8), las medicinas han salvado vidas, pero también han contribuido a terminarlas como lo demuestran, entre otros, los casos de rofecoxib (Vioxx), rosiglitazona (Avandia), y el tratamiento de reemplazo hormonal. La morbimortalidad inducida por medicamentos aprobados está en ascenso, entre otras cosas porque la sociedad está cada vez más medicalizada. Como ha comentado Gómez-Vargas (2), muchos de los ensayos clínicos no tienen el fin de descubrir nuevos productos, sino de fomentar el uso de medicamentos que no benefician al paciente, pero que pueden causarle efectos adversos más o menos serios. Así también lo sugiere Ricardo Martínez al citar a Pignarre: "la química de las moléculas 'produce fisiopatologías' con la categoría de enfermedades" (4 p.161); y, en el proceso de curar las fisiopatologías inventadas, se producen efectos adversos.

En referencia al rol de los comités de ética y la necesidad de entrenar más adecuadamente a los miembros que los integran, queremos aclarar nuestro acuerdo con la afirmación expresada por Lorenzo y Garrafa (5 p.169). Nosotros 
escribimos que sería necesario que los comités de ética fueran capaces de tamizar los ensayos clínicos de medicamentos que ofrecen terapias auténticamente innovadoras de los que solo tienen fines económicos, pero no afirmamos que los comités de ética entrenados pudieran Ilegar a hacerlo; más bien intuimos lo contrario, pero nos hubiera gustado hacer un estudio que lo demostrase. Nuestra posición (1 p.143), es que la aprobación definitiva la debería otorgar un comité nacional, tal como se hace en Brasil. En realidad, pensamos que para restaurar la credibilidad de los ensayos clínicos se requiere de instituciones independientes de la industria que se responsabilicen por el diseño, implementación y análisis de resultados de los ensayos clínicos, y que todos sean aprobados por un comité nacional y cuenten con la concurrencia de los comités institucionales. En la actualidad, hay un claro conflicto de interés, pues los resultados de los ensayos clínicos tienen un impacto económico para aquellos que los patrocinan y para los organismos que ellos contratan, incluyendo las Organizaciones de Investigación por Contrato (CROs) y los comités de ética privados.

Es importante que se fomente el debate sobre los ensayos clínicos entre los académicos, en

\section{REFERENCIAS BIBLIOGRÁFICAS}

1. Ugalde A, Homedes N. Cuatro palabras sobre ensayos clínicos: ciencia/negocio, riesgo/beneficio. Salud Colectiva. 2011;7(2):135-148.

2. Gómez-Vargas M. Dos palabras más sobre ensayos clínicos: engaño/verdad, la anemia moral de la "Big Pharma". [Debate]. Salud Colectiva. 2011;7(2):163165.

3. Forcades i Vila T. Otras cuatro palabras clave para analizar críticamente un ensayo clínico: hipótesis, realización, análisis y publicación. [Debate]. Salud Colectiva. 2011;7(2):171-173.

4. Martínez RA. Interpelando a los ensayos clínicos [Debate]. Salud Colectiva. 2011;7(2):160-162.

5. Lorenzo C, Garrafa V. Ensayos clínicos, Estado y sociedad: ¿dónde termina la ciencia y empieza el negocio? [Debate]. Salud Colectiva. 2011;7(2):166-170. la sociedad civil y en el ámbito político. Salud Colectiva ha facilitado su inicio. Los temas a debatir son múltiples, varios de los cuales han quedado enunciados en este primer esfuerzo:

- ¿Qué se debe hacer para que los participantes en ensayos entiendan las formas de consentimiento?

- ¿Qué debe/puede hacer la sociedad civil para que las agencias reguladoras prioricen la protección de los derechos humanos de los ciudadanos antes que los intereses de la industria, faciliten la investigación independiente de los ensayos clínicos y exijan transparencia?

- ¿Qué debe hacer el Estado para que las agencias reguladoras sean transparentes?

- ¿Qué deben hacer los Estados latinoamericanos para que los científicos de la región investiguen sobre medicamentos que respondan a las necesidades regionales?

- ¿Quién debe pagar a los investigadores que participan en los ensayos y a los miembros del comité de ética nacional?

- ¿Quién debe realizar los ensayos clínicos?

6. Gonorazky SE. Principio de precaución en investigación biomédica, seguridad, obligaciones post-investigación y eficacia terapéutica supuesta de las drogas experimentales. Violaciones a la dignidad de los pacientes. [Debate]. Salud Colectiva. 2011;7(2):149-151.

7. Ferrante D. Investigación clínica en América Latina y Argentina: ¿es tiempo de cambios? [Debate]. Salud Colectiva. 2011;7(2):157-159.

8. Tajer CD. Hacia la investigación clínica independiente en América Latina. Un comentario a "Cuatro palabras sobre ensayos clínicos". [Debate]. Salud Colectiva. 2011;7(2):152-156.

9. Sender H. Lines less blurred. Financial Times. 18 jul 2011:7.

10. Boletín Fármacos [Internet] c2010 [citado 27 jul 2011]. Disponible en: http://www.saludyfarmacos.org/ boletin-farmacos/

\section{FORMA DE CITAR}

Ugalde A, Homedes N. Respuesta de los autores. [Debate]. Salud Colectiva. 2011;7(2):174-176. 